\title{
Les villes du gouvernement et de l'administration sous l'empire en Hispania: questions de lectures
}

Patrick Le Roux

\section{Université de Paris 13-Villetaneuse}

leroux.patrick@club-internet.fr

Fecha recepción 22.02.2016 / Fecha aceptación 22.06.2016

\section{Résumé}

Réflexions d'ensemble sur le thème des « villes du pouvoir » et les questions historiographiques qu' il suscite aujourd' hui.

\section{Mots-clés}

Caput, Empire, Épigraphie, État, Statuts, Temporalités, Urbanisme, Ville de consommation.

\section{Abstract}

This paper provides an overview of the theme of "cities of power" and of the historiographical issues it currently raises.

\section{Key words}

Caput, Empire, Epigraphy, State, Statutes, Temporalities, Urbanism, Consumer city. 
Il y a près de quarante-ans, j' avais été convié à participer à la célébration du bimillénaire officialisé ${ }^{1}$ de la fondation de la ville de Lugo sous Auguste. La découverte récente de l' inscription de l' affranchi Saturninus ${ }^{2}$ avait attiré mon attention sur les personnels attachés à l'empereur et à l' administration provinciale à Lucus Augusti ${ }^{3}$. Le sujet pouvait paraître relativement nouveau dans le contexte de 1976 concernant les provinces Ibériques de Rome au Haut-Empire. L'enquête épigraphique était associée à une historiographie centrée sur la ville à travers les âges que les archéologues et les historiens des Antiquités commençaient aussi à explorer sous l' influence du présent : dans divers pays européens, au cours du deuxième tiers du XXe siècle, la population urbaine dépassa en pourcentage la population dite rurale ${ }^{4}$. L' urbanisation connut alors une mutation inédite qui n'a pas cessé depuis. Une économie de plus en plus fondée sur la circulation accélérée, les échanges diversifiés et incessants et sur l'essor de technologies nouvelles avait placé au cœur des agglomérations urbaines la question de leurs fonctions et de leur rayonnement ou influence sur l'environnement régional. Le fait urbain ne jouissait encore que d' un succès d'estime dans les travaux de recherche des spécialistes. Les historiens de l'Antiquité qui s'y intéressaient hésitaient entre deux orientations : l' une, qualifiée de "moderniste ", regardait la ville comme un reflet de l'État et de sa toute puissance au service de l'économie et du pouvoir à l'égal de ce qui avait été décrit par la critique libérale ou marxiste à propos de l' État moderne ; l' autre , perçue comme " primitiviste » et minoritaire, allait à la rencontre de Max Weber inventeur de la ville « consommatrice » mais aussi du penseur des relations entre les villes et les pouvoirs ${ }^{5}$. Les tendances dominantes inci-

1. La ville passait, dans l'esprit des organisateurs, pour avoir été fondée en 25 av. J.-C., ce qui n'est toujours pas démontré.

2. $A E, 1973,294$, qu' il faut rapprocher, comme l' avait fait A. Blanco Freijeiro, de $A E, 1976,312$; voir I. des Asklepieions (C. Habicht), Altert. von Pergamon (1969), VIII, 2, 88-92, nº 44. Lire également M. Christol et S. Demougin, "De Lugo à Pergame: La carrière de l' affranchi Saturninus dans l'administration impériale", MEFRA, 102, 1990, 159-211.

3. P. Le Roux, "Lucus Augusti capitale administrative au Haut-Empire", dans Actas del Coloquio internacional sobre el bimilenario de Lugo, Lugo, 1977, 83-105.

4. En France, la date affichée statistiquement est 1931 (voir le recensement de 1930 qui indique 50\% pour chacune des parties de la population). En Angleterre, le seuil fut franchi plus précocement.

5. M. Weber, La Ville, Paris, 1982 est la première traduction en langue française de Die Stadt. Voir désormais l'édition des Belles Lettres, 2013 dans la traduction également de Ph. Fritsch, révisée et annotée. 
taient à la découverte d' une histoire provinciale concernée par Rome et les indigènes à l' aune d' un concept fédérateur, celui de « romanisation » qu' il fallait toutefois définir au préalable

La notion de "villes du pouvoir ", par un glissement insensible, fait écho aujourd' hui à ce que l'on désigne, après $M$. Weber, comme un «idéaltype ». Les méthodologies ont évolué mais les modèles ne sont pas construits par chacun, pour autant, de la même manière. L' habillage n'est pas indifférent pas plus que les mots pour le dire ${ }^{7}$. Il s' agit assurément d' un thème politique dans le prolongement des réflexions sur l'Antiquité comme « civilisation du politique » et des interrogations sur l'existence ou non d' un État romain au sens plus récent de l' État institutionnel et territorial. Le mot de "capitale », en revanche, a été volontairement évité de même que le syntagme de "ville administrative » jugé sans doute, avec raison, trop restrictif et historiographiquement daté. Aborder dans un cadre provincial les «villes du pouvoir » constitue donc un changement et une source de renouvellement après cinq décennies d'enquêtes variées et de fouilles elles-mêmes mieux préservées et mieux analysées. Les questions nées d' une modification des sociétés présentes ne sauraient suffire. Les relectures du passé, plutôt que les "déconstructions ", qui semblent remiser ce qui a été pensé et écrit sans vraiment chercher à formuler une nouvelle intelligibilité historique, sont susceptibles de déboucher sur une " réinvention » historiographique, ici des « villes provinciales » et de leur rôle sous le Haut-Empire romain.

Le modèle implicite reste toujours celui de l'État-nation fondé sur le poids de « capitales » intégrées à des réseaux de pouvoir indispensables à un contrôle des territoires destiné à éviter désordres et troubles fréquents ${ }^{8}$. Les évolutions épistémologiques et idéologiques ont introduit depuis quelques décennies des discordances et des orientations historiographiques différentes et diversifiées. Les points de vue exprimés dans l'ouvrage en sont un reflet fidèle. Trois temps forts se dégagent : l'esprit même de l'administration romaine et ses caractères dans le contexte de l'empire et du regard romain. L'espace, ses constructions territorialisées ou non, les structures qui en sous-tendaient l'organisation et le fonctionnement sont mis en exergue. Le deuxième moment relève des définitions mêmes des villes et de leur inclusion dans des réseaux hiérarchisés ou non. Leur rayonnement et les expressions symboliques qui en émanent rythment la question de l'emprise sur les populations environnantes et appellent une réflexion sur les temporalités. Sans une exposition préalable concernant les documents, les méthodes, les concepts et les modèles, bref les orientations intellectuelles et épistémolo-

6. P. Le Roux, A. Tranoy, "Rome et les indigènes dans le Nord-Ouest de la péninsule Ibérique. Problèmes d'épigraphie et d' histoire", $M C V, 9,1973,177-231$. Le concept de "romanisation» commençait à faire l'objet de renouvellements au profit d' «acculturation» et d' «intégration» voire de «coexistence».

7. L'enjeu est évidemment la question de la domination romaine sur laquelle il n'est pas nécessaire d' insister à ce stade de la réflexion.

8. Lire M. D. Dopico Caínzos, "Nuevas formas de administración, nuevas formas de control: la fundación de Lucus Augusti y su conventus", Portugalia, 34, 2013, 83-100, qui, dans l' esprit des "Ciudades del poder» fonde la réflexion sur l'existence d'une volonté étatique du pouvoir et sur la concentration en un lieu privilégié de fonctions administratives source d' unification et de cohésion des conventus juridiques autour de la ville capitale. 
giques à l'origine des diverses lectures et interprétations il n'y a pas de mesure objective et efficace des résultats énoncés.

\section{Méthodes et débats}

L'érudition est au départ. Elle n' informe que si on l' interroge. Dans les disciplines de l'Antiquité, elle est aléatoire et lacunaire. Support indispensable, elle ne conduit à l' intelligence historique que par des méthodes et des analyses multipliées, aucune interprétation ne découlant directement d'un raisonnement aussi adapté soit-il à la recherche. Les documents, par leur apport factuel et discursif, ouvrent seulement des portes. Il est rare qu' ils proposent une réponse complète et satisfaisante sauf à s'en tenir à un système de lecture qu' il faudrait considérer comme plus scientifique par définition qu' un autre de même qualité. Nos disciplines ne sont pas des sciences exactes, ce qui est une incitation supplémentaire, intellectuellement fructueuse, à la recherche de concepts pertinents.

Incontestablement, la documentation relative aux « villes du pouvoir» ne cesse de s'enrichir, de s'étoffer et de progresser à force d'être mieux approfondie. C'est vrai aussi, malgré ce que l'on entend parfois, des textes à caractère littéraire qu' il s' agisse de Strabon, de Pline l'Ancien, de Tacite, de Dion Cassius et de beaucoup d' autres qui offrent en permanence des occasions de découvertes et de révision, même sans le secours du présent et du fonctionnement actualisé des disciplines historiques. Il est non moins vrai que les inscriptions jouent un rôle essentiel. L' attente les concernant s'avère même forte et certainement trop forte, ce qui engendre parfois des effets pervers, que ce soit en termes de falsifications ou de mauvaise appréciation du document lui-même susceptible d' induire les lecteurs en erreur ou d'accroître illégitimement la suspicion. Il convient de rappeler que les documents épigraphiques de contenu renouvelé sont souvent lacunaires et fragmentaires ou privés du contexte qui permettrait d'emblée d'en mesurer la fiabilité et la portée avec la précision souhaitable. En outre, un texte gravé sur la pierre ou le métal n'est plus considéré comme un témoin primaire informant directement sur le passé. Le message qu' il comporte n' est ni objectif ni neutre : il est dépendant d' un monument projeté, d' un environnement choisi ou défini, et d' une expression sociale qui en déterminent la signification exacte. Il faut donc ajouter que la logique interne d' une inscription ne fournit pas immédiatement un raisonnement tout prêt sur l' insertion des informations dans un ensemble plus ou moins connu et connaissable.

La recherche sur les villes n'est pas imaginable sans le recours aux fouilles et aux études archéologiques. Dans ce cadre, les méthodologies utilisées, les critères chronologiques en grande partie fondés sur la seule céramique en fonction d' un repère extérieur censé caler les datations sont souvent fragiles et entachés de subjectivité et de conviction malgré ce qui est affirmé. Le recours aux apports de la numismatique offre des éléments non négligeables mais les incertitudes concernant les stratigraphies et la circulation des monnaies contraignent à de la prudence et conseillent parfois le doute méthodique. Les plans, les architectures, les phases de construction et d'arrêt temporaire plus ou moins long sont surtout source de comparaisons souvent orientées par des schémas commodes mais simplificateurs et limitées dans leur éventail, par la force des choses. Comme il a été souligné à plusieurs reprises cependant, la 
mise en place d' un urbanisme renouvelé et monumental est une donnée à prendre sérieusement en compte à la fois en relation avec l' histoire locale et avec les évolutions historiques plus globales. Sous cet angle, les risques de déformation sont permanents et les conclusions doivent être constamment confrontées à d' autres paramètres. L'existence d' une ville archéologiquement conservée ne dit rien par elle-même ${ }^{9}$. Les techniques, les styles, les choix fondamentaux n'obéissaient, localement, à aucune obligation, à aucune sollicitation autre que financière. Pour le reste, les commanditaires et les spécialistes qu' ils utilisaient décidaient au cas par cas et à chaque étape d' une mise en place qui s'étendait sur plusieurs décennies.

Les documents sont inséparables d'objets méthodologiques construits en rapport avec des contenus proposés par les « sciences » humaines et sociales. Les « villes du pouvoir » sont en ce sens tributaires de conceptions géographiques, juridiques, politiques, économiques et culturelles à confronter aux expériences antiques. Les mots sont les outils les plus précieux des historiens, ce qui ne veut pas dire que le récit ou la réflexion historique soient condamnés à n'être qu' un discours. Cela signifie seulement que les mots engagent la construction du réel et du passé dans ce qu' elle a de concret, de perceptible et d' intelligible : il est nécessaire d'en prendre la mesure et d'en retrouver les sens cachés en fonction de contextes linguistiques et sociaux rigoureusement circonscrits. L' État n' a pas toujours été. Recourir à son usage oblige à prendre des distances avec une conception «évidente " qui n'a pas de fondement avéré surtout dans un contexte de l'Antiquité. Sur le plan du langage, il n'est pas possible de faire l' impasse sur l'absence de l'expression caput conventus. Pour un regard romain et administratif ce n'était pas la raison d'être de villes telles que Bracara, Lucus ou Asturica. Il faut aller plus loin. Il n'est, dans cette direction, ni nécessaire ni légitime de faire comme si les villes ayant donné leur nom aux conventus désignés par Pline et les inscriptions avaient joué le rôle de centre administratif de la production des bornes milliaires et contrôlé on ne sait quel réseau routier limité au conventus ${ }^{10}$. Il en est de même pour le syntagme caput provinciae qui n'est pas utilisé et n'aurait pas le sens qui lui est attribué au sein d'une hiérarchie urbaine supposée ${ }^{11}$.

9. B. Goffaux, "Promotions juridiques et monumentalisation des cités hispano-romaines", Salduvie, 3, 2003, 143-161.

10. Le silence des inscriptions routières sur ce point aurait dû suffire. Le débat ouvert il y a quelques décennies ne mérite pas que l'on y revienne car il n'a débouché sur rien de concret : voir déjà les critiques adressées à l'article de M. D. Estefanía Álvarez, "Notas para la delimitación de los conventus jurídicos en Hispania“, Zephyrus, 9, 1958, 51-57. Outre des questions de tracé non avéré ou discuté, la méthode met en cause un regard rétrospectif emprunté aux époques contemporaines dont l'esprit n'est pas applicable aux questions routières ni au conventus s'agissant de l'Antiquité romaine, malgré une opinion admise sans examen autre par les archéologues spécialisés. La loi de la colonie Genetiva Iulia Vrsonensium LXXIIX ou le Digeste XLIII ne suggèrent que des responsabilités des riverains dans l'entretien des voies publiques ce qui n'entraîne aucune conséquence connue sur l'organisation globale du réseau. Ces dispositions n'ont pas plus d' incidence sur les formes d'administration et de contrôle que n'en ont pour l'ensemble de l'armée romaine celles qui concernent à Vrso la défense de la cité (CIII).

11. P. Arnaud, “Titulatures municipales et réseaux urbains : le titre de métropole dans les provinces romaines d'Orient”, Cahiers de la Méditerranée, 64, 2002, 39-47. 
Il s'ensuit que la première thématique reconnaissable au fur et à mesure des explorations du thème est relative à la notion même de « territoire » dont l' utilisation est particulièrement délicate et complexe et ne va pas de soi.

\section{II.- L' Empire et les provinces : de la notion de «territoire »}

La question méthodologique centrale de la relation existant entre les réalités présentes et les faits passés est apparue de manière claire s'agissant des modalités de domination et de contrôle des provinces et des espaces provinciaux. Chacun ne pose pas les problèmes dans les mêmes termes en fonction de choix historiographiques inévitables à partir du moment où la documentation, lacunaire et laconique, n' apporte pas de réponses susceptibles de recueillir l' unanimité. Toutefois, certaines limites sont aisées à établir en fonction de ce que nous pouvons lire dans les documents antiques et des vocabulaires et impressions qu' ils véhiculent. La première et la plus importante est que les pouvoirs romains ne se réfèrent pas à des territoires tels que les États modernes nous y ont habitués : ce qui importe ce sont les habitants, les populations et non des abstractions. Les cités sont des communautés de citoyens locaux comme l'exprime l' usage normal en épigraphie de l'adjectif géographique : au hasard, Tarraconenses, Iulienses, Valentini, Bracaraugustani etc. sous la forme du génitif pluriel s' il est précédé de civitas, res publica, colonia ou municipium. L'empire romain lui-même n'avait pas d'autre appellation que celle-là (imperium romanum ou populi Romani) ${ }^{12}$ ce qui veut dire qu' il n'était pas une entité identifiable par un seul mot géographique ou ethnique à la différence de nos États ou Nations modernes. La " territorialité » de l'empire, des provinces et des cités ne résultait pas d' une définition juridique mais d' une nécessité pratique et fiscale, le mot territorium ayant un sens judiciaire à en juger d' après le Digeste ${ }^{13}$.

Dans ce contexte que l'on ne peut pas ignorer, le conventus n' est qu' une circonscription arbitrairement délimitée, conséquence d' une méthode comptable ou arithmétique à l' aune des recensements et du nombre des cités, rendue nécessaire par l'exercice de la juridiction indépendamment de la qualité et de la quantité des magistrats désignés pour faire face aux tâches qu'elle impliquait ${ }^{14}$. Asturica n' a d'ailleurs pas donné son nom au conventus Astu-

12. Surtout pas « romanitas » qui n'est utilisé, sans lendemain, que par Tertullien dans le de Pallio, 4.1, avec un sens culturel dans le contexte d' une adresse aux citoyens de Carthage.

13. Territorium d'après Pomponius est crédité d' une étymologie peu sûre en relation avec terreo et le pouvoir d'effrayer dont disposait un magistrat. D' autres témoignages préfèrent insister sur terra et le fait de fouler la terre, ce qui en souligne le caractère concret : c'est cependant ager qui est utilisé normalement et désigne les terres cultivables et pâtures servant à la subsistance de la communauté.

14. Les formulations de Pline l'Ancien sont éclairantes sur la définition même du conventus : 3.23 : Tarracone disceptant populi XLII ; 3.24 : Caesaraugusta ... recepit populos LV; 3.25 : Carthaginem conveniunt populi LXV; 3.26 : In Cluniensem conventum Varduli ducunt populos XIIII; 3. 28 : Lucensis conventus populorum est sedecim. Les populi sont la référence fondamentale et forment la base statistique des conventus dont la ville éponyme n'est qu' un pôle d' attraction ou de mise en mouvement. La relation est physique plus qu' administrative et n'est que judiciaire quoi qu' il en soit. 
rum ce qui montre que la ville n'était qu' un lieu de réunion pour ceux qui devaient plaider une cause en présence d' un détenteur d' un imperium. La création et l'essor d'Asturica Augusta suggèrent également que de nombreuses circonstances autres que le conventus y ont contribué. Nous ignorons jusqu'à quel point les décisions impériales ont influé sur le développement monumental et urbain de la ville, comme nous ne savons qu' indirectement que le pouvoir fit en sorte de favoriser, dans une mesure que l'on ne peut pas évaluer sérieusement, le succès de Bracara Augusta et de Lucus Augusti. Enfin, Asturica est devenue le siège du procurateur d'Asturie-Galice dont la présence et l' administration pesèrent certainement plus que l'identité de siège des assises judiciaires sur son rayonnement et son urbanisation, ce que reflète le qualificatif d' Vrbs magnifica de Pline à son propos ${ }^{15}$. Au départ, Rome évaluait la situation en fonction du recours à la force armée ce qui ne correspondait pas à une stratégie au sens moderne du mot. L'attitude des populations, leur imprévisibilité estimée, les effets de comportements violents sur l'environnement humain immédiat constituaient ce que j' appellerais le « regard militaire » du pouvoir romain. De là, la décision d' intervenir ou non, d'attendre ou de prendre des dispositions exceptionnelles sachant que le moindre peuple turbulent comptait autant qu' un roi hellénistique ou que Carthage.

Avec les victoires et l'expansion du dominium romain, le regard s'est apaisé puis pacifié sans cesser d'être vigilant. Les Hispaniae ont peu à peu intégré le monde contrôlable et contrôlé en totalité par l' inclusion des terres encore mal connues : celles surtout des Astures et des Cantabres remémorant parfois les "sauvages " Celtibères. Autant que l'on puisse en juger, il n'y eut pas pourtant de rupture profonde dans les méthodes de gouvernement et de l'exercice du pouvoir. Les populations reconnues comme constituant des communautés étaient censées entretenir des relations bilatérales régulières avec Rome identifée à l'empereur et à l'empire. Au lieu d'armées hostiles, l' autorité romaine avait affaire à des communautés gouvernées par des familles influentes localement et capables d'assurer la transition nécessaire. Contrairement à ce qui a été longtemps dit, aucune hiérarchisation territoriale à caractère administratif n' a alors vu le jour ${ }^{16}$. La province, la cité, les conventus, les circonscriptions financières ne formaient pas un ensemble emboité et cohérent régi par on ne sait quelle centralisation rationnellement ordonnée conduisant à Rome. À l' inverse, on ne décèle pas de volonté décentralisatrice qui fût différente de l' autonomie locale, socle de l'organisation de l'empire. Sous cet angle, le conventus ne peut pas avoir été un échelon intermédiaire entre la cité et la province. Les représentants du pouvoir se déplaçaient s' il le fallait. Il n’y avait aucun magistrat ni titulaire dépendant de l'empereur à la tête d' un conventus dont les archives voyageaient avec le responsable de la justice. Les tabularia ne concernaient pas le conventus mais l' administration financière. Une province romaine n'était pas un État bureaucratique dont les conventus auraient été les relais tatillons et soupçonneux.

Les capitales provinciales n'ont acquis cette identité qu' avec beaucoup de temps. Là aussi Pline nous invite à prendre les données avec simplicité. Jamais il ne désigne Cordoue

15. Pline l'Ancien, NH. 3.28.

16. La hiérarchie est alors celle des statuts politiques de définition complexe non pas celle de régions géographiques inexistantes en tant que telles.

Revista de Historiografía 25, 2016, pp. 17-27 
ou Tarraco comme capitales provinciales dans son inventaire du livre III. L'empire n'a qu' un caput, Rome à la tête de l' Italie. Les villes de résidence d' un gouverneur ne méritaient pour appellation que celle de sedes legati ou proconsulis sachant que Galba, selon Suétone, se partageait encore entre Carthagène et Tarragone. Les villes du pouvoir par excellence étaient surtout les colonies. L'épisode de L. Caecilius L. f. Pap. Optatus décidant de gratifier Tarragone de ses générosités testamentaires si Barcino ne respectait pas ses volontés ne se comprend que comme la mise en concurrence de deux colonies romaines prestigieuses et voisines et non comme un hommage singulier à la capitale provinciale, illogique en ce cas dans la mesure où la supériorité affichée de Tarragone aurait flatté plus qu' affecté Barcelone. Les politiques provinciales visant à enregistrer les doléances d' une province et à engager des réformes d' intérêt général ne sont pas non plus attestées. Le concile provincial n' avait ni cette vocation ni cette raison d'être, le culte impérial l'emportant ici et avec lui la possibilité de confirmer la fides des cités envers le pouvoir. Les relations n'étaient ni codifiées strictement ni limitées autrement que par le recours qu' y consentait l'empire. Le meilleur symbole des différences avec nos critères actuels est sans doute la notion même de «territoire » des cités dont on constate qu' ils étaient bornés. Pour autant, une cité n'était ni souveraine ni indépendante et son pouvoir de contrôle était compris dans des « limites » qui n'étaient pas des «frontières ». Les critères fiscaux et judiciaires en rendaient aisément compte.

Il est possible d'ajouter d'autres arguments tirés de l'absence de réseaux, de la notion même de citoyenneté éloignée de la nôtre. Le gouvernement établissait des relations, qui une fois nouées, ne pouvaient plus, au nom de la fides, être mises en cause ni récusées. L'administration quant à elle se fondait non sur des territoires et des entités abstraites mais sur des populations et des documents chiffrés dont on admettra qu' ils n'étaient pas toujours rigoureux ni tenus régulièrement à jour, ce qui n' est pas un indice de " primitivisme » comme on le sait aussi aujourd' hui.

\section{III.- Les villes et les regards de Rome}

La conception romaine de la vie policée et civilisée était héritée d' une longue tradition, surtout grecque en ce cas mais pas uniquement. La ville était donc un reflet et un ferment de relations plus sereines en même temps qu' un symbole d' un état politique aux antipodes de la " barbarie ». Rome était une ville et en avait fondé très tôt. Les Phéniciens, les Puniques et les Carthaginois l'avaient précédée. Il a fallu du temps et les urbanisations romaines successives ont évolué avant d'acquérir une structure extensible et modélisable sous l' impulsion d' un pouvoir renouvelé.

On observe aisément que les villes créées au nom de César ou d'Auguste ou héritières d' un passé favorable à leur illustration, et parfois même refondées, sont devenues des villes souvent plus importantes que les autres à l'échelle provinciale. On se gardera d'établir une loi car chaque communauté urbaine a connu un destin différent. Les colonies césariennes et augustéennes ont en grande partie pu s'épanouir. Plusieurs éléments sont à considérer pour l'expliquer. Leurs installations, le plus souvent à l' aide de vétérans ou de populations regroupées en ce lieu, ont bénéficié de l'attention d' un pouvoir monarchique attentif à leur succès 
social et monumental, notamment dans le cas des colonies, et ont coïncidé avec une période de paix nouvelle incitant à construire et à bâtir pour la renommée. Il y a dans cette manière de dire une nuance importante avec la formule retenue pour Caesarea de Maurétanie devenue à l' image de Berlin-Ouest au temps de l' après-guerre une "ville vitrine ${ }^{17}$. La transposition fleure la formule rhétorique et le contexte est trop étranger à la situation de l' Empire romain d'Auguste pour que la comparaison soit recevable. Intellectuellement, elle soulève une interrogation fondamentale aussi pour la compréhension de l' urbanisation des époques romaines et singulièrement impériale. Y a-t-il une lecture généralisable et déchiffrable des développements urbains dans les provinces romaines et à quelle condition?

Concrètement et symboliquement une ville n'était pas définissable a priori par des fonctions supérieures ou non exprimant on ne sait quel pouvoir particulier. Elle était une agglomération plus ou moins remarquable selon ses monuments, leur nombre et leur beauté. Elle traduisait l'existence d' un pouvoir coiffant la population et assurant une autorité exprimée au nom de l'ensemble des citoyens capables de se nourrir et de commercer. L'organisation de l'empire n'a pas mis fin à l' idéal d' autonomie et d'autarcie des cités, ce qu'étaient au premier chef les colonies ou villes dotées d' un rôle administratif du fait de l'accueil de tel ou tel représentant de Rome. La ville qualifiée d'administrative ne se revendique jamais comme telle, même si la fonction pouvait parfois contribuer à son prestige. L' importance des familles dirigeantes, leur illustration et leurs générosités recevaient certainement plus d'écho et signifiait que ces élites locales assuraient richesse et éclat à la ville. Tarragone et Cordoue étaient comme Astigi, Mérida ou Carthagène des colonies romaines. Rien ne permet de conclure avec certitude que le choix de la résidence d' un gouverneur ou d' un procurateur ou autre légat juridique ait été vraiment à l'origine de leur croissance ou « réussite » et du développement de leur population au-dessus de la moyenne d' autres colonies.

La situation géographique, les contextes culturels, politiques et les circonstances ne sauraient être oubliés dans cette histoire urbaine. Il a, encore une fois, fallu du temps et le temps a introduit des modalités et des rythmes singuliers que l'on définira comme des «temporalités » variées et concurrentes. Ni l' administration, ni les événements, ni la politique, ni l' histoire sociale, ni l'économie, ni les faits culturels n' habillent l' histoire ni ne confèrent un sens déterminant à eux seuls aux structures historiques qui expliqueraient des périodisations qu' un examen attentif rend nécessairement caduques. Quand ce ne sont pas les gouvernants eux-mêmes par leur mobilité, leurs résidences multiples, leurs voyages ou déplacements faits de choix arbitraires ou d' ignorances volontaires qui ont créé les conditions d'évolutions, au sens le plus neutre, et de changement de visibilité des villes, les événements militaires imprévisibles au départ ont eu pour effet $d$ ' attribuer un rôle politique et administratif nouveau à certaines agglomérations. Dans les provinces Ibériques, ce n'est pas avant le Ve siècle qu' un phénomène de cette nature fut perceptible.

17. Il ne s' agit pas ici de remettre en cause l' importance de la monographie pionnière sur de nombreux plans de Ph. Leveau, Caesarea de Maurétanie. Une ville romaine et ses campagnes, Rome 1984. La question soulevée est historiographique et non à proprement parler méthodologique. 
Il est donc difficile d'attribuer aux villes provinciales romaines des « spécialisations » qu' il s' agisse de "capitale ", de présence militaire, de production ou d'échanges, de dimension religieuse. La ville de garnison n'existait pas et la transformation de Legio VII Gemina dans l'Antiquité Tardive ne promut pas vraiment un nouveau type de ville. La ville religieuse n' avait pas non plus d'existence séparée. L'acte religieux accompagnait la vie publique et les décisions nécessitant l'accord des dieux. Aucune ville-sanctuaire ou ville appelée plus tard « de pèlerinage » ne peut être identifiée dans les provinces Ibériques de Rome, ce qui n'exclut pas l'existence de sanctuaires ayant une renommée régionale ou provinciale, voire plus encore, et un rayonnement particulier. Il semble plus fructueux de ne pas enfermer les villes, grandes et petites, dans des catégories étrangères aux regards " romains ». Les relations urbaines et leur caractère plus ou moins ouvert peuvent aider à situer le rôle et l' influence d' une ville dans un contexte provincial ou régional. Outre les insuffisances des témoignages disponibles, même sur le plan archéologique, il n' y a pas de lignes directrices claires sur un plan économique, administratif, religieux ou politique qui tracent des orientations recevables et propres à telle ou telle région, sauf à transposer des modèles actuels qui peuvent créer l' illusion de continuités entre le passé et le présent.

Les «villes du pouvoir " parlent à première vue de "visibilité » urbaine dont l'émergence n'a de réalité pour nous aujourd' hui que du point de vue de Rome et des citoyens locaux attachés à répondre à ses sollicitations et à asseoir leur propre prestige local. Bien sûr, les aspects économiques et le fonctionnement même des économies urbaines explicitent, en les diversifiant, les développements urbains inégaux. Comme il a été remarqué depuis longtemps, les contenus divers des villes et de leurs fonctions supposées ont " résisté " aux mutations pendant les longs siècles de l'empire pour des motifs liés à la place de la cité quelle qu' elle fût dans l'organisation impériale.

La richesse d' un thème historiographiquement mis à jour et la diversité de son traitement parlent d'eux-mêmes. Il n'était pas possible d' insister longuement sur le culte impérial, sur les cultes orientaux, sur l' image plus ou moins attrayante des villes. Ces données n' appartiennent pas seulement à l' histoire des "villes du gouvernement ", à plus forte raison dans un cadre provincial. En ce sens, la catégorie mise en exergue oriente principalement vers les rapports entre statut juridique, importance politique, urbanisation et urbanisme et présence d' institutions administratives au demeurant difficiles à identifier archéologiquement et concrètement. Un clivage apparaît, bien connu des historiens du monde romain : celui des poids respectifs et concurrents ou non des regards " militaires » et des regards " pacifiés » de Rome. Il n' y a pas eu là non plus d'élaboration de stratégies ou militaires ou administratives définissables. Plus que les transformations sociales, enfin, la question sous-jacente a été celle de la "provincia» et de ce que peut être ou ne peut pas être une définition de l' Empire romain, si tant est qu' il faille le définir.

On l'a entendu. Il n' est ni possible ni souhaitable que tout le monde parle d' une seule voix sur ces sujets à partir du moment où les interrogations sont justifiées par une méthode définie. Aujourd' hui, l' historiographie commence à entrer dans la fabrication de l' histoire afin de mieux délimiter les écritures, les récits, les discours auxquels elle donne lieu. L' une des tâches nouvelles est donc d' inventer des concepts qui ne soient empruntés, dans la mesure du possible, qu' aux données antiques ou mieux aux formations politiques et sociales antiques. 
En guise d'exemple, réfléchissons au sens des dynamiques observables. Il ne s'est pas agi du passage d' une action pragmatique élaborée par le sénat à une structure administrative bien réglée et ordonnée adaptée à une vision " territoriale », voire « territorialisée », des provinces. Comme je l' ai déjà suggéré, la ligne directrice des changements relève d' une activité appuyée au moins en partie sur l'armée à une organisation plus civile et soucieuse de favoriser un mieux « gouverner» (terme à bien définir), sans que la conception même qu'eut Rome de son pouvoir et de son contrôle ait beaucoup changé. L'exotisme de Rome comme traduction du passé est la condition préalable pour bien saisir rapprochements et éloignements. Il convient donc pour ne pas prolonger davantage le propos de souligner la nécessité d' utiliser le pluriel, comme le fait le programme, et de parler des « villes de gouvernement» ou des « villes du pouvoir » sans référence à un système construit à l' avance. 